\title{
Equation of state of strongly interacting matter: spectra for thermal particles and intensity correlation of thermal photons
}

\author{
Somnath Det, Dinesh K. Srivastava $\$$, and Rupa Chatterjee \\ Variable Energy Cyclotron Centre, 1/AF Bidhan Nagar, Kolkata 700 064, India
}

\begin{abstract}
We find that an equation of state for hot hadronic matter consisting of all baryons having $M<2 \mathrm{GeV}$ and all mesons having $M<1.5 \mathrm{GeV}$, along with Hagedorn resonances in thermal and chemical equilibrium, matches rather smoothly with lattice equation of state (p4 action, $N_{\tau}=8$ ) for $\mathrm{T}$ up to $\approx 200 \mathrm{MeV}$, when corrected for the finite volume of hadrons.

Next we construct two equations of state for strongly interacting matter; one, HHL, in which the above is matched to the lattice equation of state at $T=165 \mathrm{MeV}$ and the other, HHB, where we match it to a bag model equation of state with critical temperature $T_{c}=165 \mathrm{MeV}$. We compare particle spectra, thermal photon spectra and histories of evolution of the quark-gluon plasma produced in the central collision of gold (lead) nuclei at RHIC (LHC) energies, considering ideal hydrodynamical expansion of the system. The particle and thermal photon spectra are seen to differ only marginally, for the two equations of state. The history of evolution shows differences in the evolution of temperature and radial velocity, as one might expect.

We calculate intensity interferometry of thermal photons and find it to be quite distinct for the two equations of state, especially for the outward correlation. The longitudinal correlation also shows a dependence on the equation of state, though, to a smaller extent.
\end{abstract}

Keywords: EOS, QGP, lattice, interferometry, particle spectra.

$\ddagger$ somnathde@veccal.ernet.in

$\S$ dinesh@veccal.ernet.in

|| rupa@veccal.ernet.in, Present address: Department of Physics, University of Jyväskyl; Jyväskylä, Finland 


\section{Introduction}

The primary goal of colliding heavy nuclei at relativistic energies is to study the behaviour of quantum chromodynamics (QCD) at high energy density. Relentless theoretical efforts over more than two decades have given rise to a description where the two Lorentz contracted nuclei pass through each other and multiple parton-parton scatterings and a vehement production of partons take place. This may lead to a deconfined state of quarks and gluons in local thermal equilibrium, called quark-gluon plasma (QGP). The unique importance of these studies lies in the well accepted premise that the early universe was in the form of QGP at around a few microseconds after the Big Bang. Recent experiments at the Relativistic Heavy Ion Collider (RHIC) at Brookhaven National Laboratory have provided a clear proof of the formation of QGP [1] or rather a strongly interacting QGP (sQGP), which behaves almost like an ideal fluid [2]. Notable confirmations include observations of jet quenching [3, 4], elliptic flow [5, 6], recombination of partons as a process of hadronization [7], and electromagnetic radiations [8]. The theoretical descriptions include parton-cascade model, which represents the multiple collisions and multiplication of the cascading partons using a transport theory [9]. This has been supplemented with a hadronic cascade [10], once the density of partons is sufficiently small. The initial state has also been modelled as a colour gluon condensate [11].

If a quark gluon plasma is formed in such collisions at some initial time, then assuming a continued thermal and chemical equilibrium, the entire evolution of the system can be calculated using relativistic hydrodynamics [12]. The condition of chemical equilibrium has been relaxed in several studies, with interesting results [13, 14, 15]. The consequence of viscosity, in the QGP or in the hadronic phase, is also a subject of considerable interest [16].

Our goal in the present study is rather modest but well defined. We consider that relativistic collision of heavy nuclei (at RHIC and LHC energies) leads to formation of quark gluon plasma at time $\tau_{0}$. The plasma then expands, cools, and hadronizes either in a rapid cross-over as suggested by lattice QCD calculations or in a first order phase transition at $T=T_{c}$, used in a large number of calculations over the last two decades using a bag model equation of state (EOS). We model the hadronic phase as consisting of all mesons having $M<1.5 \mathrm{GeV}$ and all baryons having $M<2 \mathrm{GeV}$ along with or without Hagedorn resonances.

We find that the inclusion of Hagedorn resonances and the finite volume for the hadrons leads to thermodynamic quantities which join rather smoothly to those from a lattice equation of state ( $\mathrm{p} 4$ action; $N_{\tau}=8$ ) [17] for zero baryonic chemical potential. We get two different equations of state by switching over from this hadronic state to either the lattice equation of state (HHL) or to a bag model equation of state (HHB) at $T=165 \mathrm{MeV}$. The bag model EOS admits a mixed phase at $T=165 \mathrm{MeV}$. The lattice equation of state, on the other hand, displays a sharp cross-over for $180<T<190$ $\mathrm{MeV}$. 
We calculate thermal particle production, thermal photon production and the history of evolution of the system using the two equations of state HHL and HHB for central collision of gold (lead) nuclei at the highest RHIC (LHC) energy. The particle and photon spectra are seen to be only marginally dependent on the EOS.

The history of evolution shows noticeable differences for temperature and radial velocity as one might expect. Recalling that the intensity interferometry of thermal photons is sensitive to the history of evolution of the system, we look at the outward, side-ward, and longitudinal intensity correlation of thermal photons having $K_{T} \leq 2 \mathrm{GeV}$. Again we find only a marginal difference in side-ward and longitudinal correlations.

The outward correlation of thermal photons is seen to clearly distinguish between the two equations of state.

The paper is organized as follows. In the next section we discuss the construction of the two equations of state HHL and HHB. In section III, the initial conditions and the results for the particle and thermal photon spectra at RHIC and LHC energies are given. In section IV, we discuss the history of evolution of the system for the two equations of state; as well as the source-function for the production of photons. In section $\mathrm{V}$, we give our results for intensity interferometry of thermal photons. Finally we summarize our findings in section VI.

\section{Equation of State}

The resonance gas model relies upon the equivalence of thermodynamic properties of an interacting gas of hadrons and a free gas of hadrons and their resonances [18].

We consider a hadron resonance gas, which consists of all mesons with mass $<1.5$ $\mathrm{GeV}$, and all baryons with mass $<2.0 \mathrm{GeV}$ and their antiparticles. We include Hagedorn states [19] having $M>2 \mathrm{GeV}$. Thus mass spectrum can be written as

$$
\rho(m)=\rho_{\mathrm{HG}}(m)+\rho_{\mathrm{HS}}(m),
$$

where

$$
\rho_{\mathrm{HG}}=\sum_{i} g_{i} \delta\left(m-m_{i}\right) .
$$

In the above, the sum runs over all the discrete hadronic states and their corresponding degeneracies are given by $g_{i}$. All the results discussed in this work assume zero baryonic chemical potential $\left(\mu_{B}=0\right)$, which is quite reasonable for top RHIC and LHC energies.

We take the density of Hagedorn states from Ref. [20]:

$$
\rho_{\mathrm{HS}}(m)=A \frac{\exp \left(m / T_{H}\right)}{\left(m^{2}+m_{0}^{2}\right)^{5 / 4}},
$$

where, $A=0.5 \mathrm{GeV}^{3 / 2}, m_{0}=0.5 \mathrm{GeV}, T_{H}=0.196 \mathrm{GeV}$, and $m$ varies from $M_{0}=2$ $\mathrm{GeV}$ to $M_{\mathrm{Max}}=12 \mathrm{GeV}$. It is noted that the inclusion of the Hagedorn resonances is responsible for the rapid chemical equilibration of hadrons produced in relativistic heavy ion collisions [20]. This may also signal existence of actual hadronic states (see, e.g., Ref. [21, 22, 23]) which have not yet been clearly identified. Only mesonic Hagedorn 
states are considered (as in the above studies) and various thermodynamic quantities like - pressure, energy density, number density, and entropy density of the hadronic gas are calculated using standard methods of statistical mechanics.

Several procedures [24, 25, 26, 27] have been used in the literature to account for the finite volume occupied by the hadrons. The treatment advocated by Kapusta and Olive [27] is believed to be thermodynamically consistent and has been used for this study. We shall see that this correction plays an important role in getting results in conformity with those from lattice calculations. According to this treatment, the finite or excluded volume (xv) corrected pressure, temperature, energy density, and entropy density are related to those for the point-particle (pt) values as:

$$
\begin{aligned}
& p_{\mathrm{xv}}=\frac{p_{\mathrm{pt}}\left(T^{*}\right)}{1-\frac{p_{\mathrm{pt}}\left(T^{*}\right)}{4 B}}, \\
& T_{\mathrm{xv}}=\frac{T^{*}}{1-\frac{p_{\mathrm{pt}}\left(T^{*}\right)}{4 B}}, \\
& \varepsilon_{\mathrm{xv}}=\frac{\varepsilon_{\mathrm{pt}}\left(T^{*}\right)}{1+\frac{\varepsilon_{\mathrm{pt}}\left(T^{*}\right)}{4 B}},
\end{aligned}
$$

and

$$
s_{\mathrm{xv}}=\frac{s_{\mathrm{pt}}\left(T^{*}\right)}{1+\frac{\varepsilon_{\mathrm{pt}}\left(T^{*}\right)}{4 B}},
$$

where $T^{*}$ is the temperature for the system having point particles and $B$ (taken as $B^{1 / 4}=340 \mathrm{MeV}$ from Ref. [20]) is the bag constant in the MIT Bag model description of hadrons.

We discuss our results for the hadronic matter with successively increasing richness of the description; viz., hadron gas, volume corrected hadron gas, hadron + Hagedorn gas, and volume corrected hadron + Hagedorn gas.

The action for the lattice calculations is given by:

$$
\frac{\Theta^{\mu \mu}(T)}{T^{4}} \equiv \frac{\varepsilon-3 p}{T^{4}}=T \frac{\partial}{\partial T}\left(p / T^{4}\right) .
$$

The parametrized form of $\Theta^{\mu \mu}(T) / T^{4}$ is taken from Ref. [17], where simulation results are given upto a temperature of $539 \mathrm{MeV}$. It is arbitrarily extended to larger values of temperature in our calculations for LHC energy.

Thus, the pressure is obtained by integrating $\Theta^{\mu \mu} / T^{5}$ over the temperature,

$$
\frac{p(T)}{T^{4}}-\frac{p\left(T_{0}\right)}{T_{0}^{4}}=\int_{T_{0}}^{T} d T^{\prime} \frac{1}{T^{5}} \Theta^{\mu \mu}\left(T^{\prime}\right) .
$$

It is found that the pressures for all the four descriptions of the hadronic matter discussed earlier, are nearly identical at a temperature of about $140 \mathrm{MeV}$. Thus, for lattice results the final values for the pressure are obtained by taking $T_{0}$ as $140 \mathrm{MeV}$, and adding the corresponding pressure from the hadronic matter calculations.

In Fig.1 (a-f) our results for the energy density, pressure, and entropy density are shown. 
We see that the energy density, pressure, and the entropy density for the hadronic gas (point particles) rise rapidly as the temperature increases beyond $160 \mathrm{MeV}$, as the number density of the particles becomes large. Switching on the volume corrections, arrests this rapid rise, and gives results which approach the values obtained from lattice calculations.

Inclusion of Hagedorn resonances gives results which are similar in shape to those from lattice calculations over several tens of $\mathrm{MeV}$. Best agreement with the lattice calculations are obtained when the hadron + Hagedorn gas is corrected for the finite volume of the particles. Now we see a very close agreement of all the thermodynamic quantities for the hadronic matter with those obtained using the lattice calculations.

In view of the above, in the following we shall describe the hadronic matter as volume corrected hadron + Hagedorn gas.

The results for the square of speed of sound for the four descriptions of the hadronic matter and their comparison with the one obtained from the lattice calculations are shown in Fig. 2, Once again we see that the hadron + Hagedorn gas with volume correction gives a speed of sound which is close to that for the lattice calculations over a large window of temperature.

Now we construct two equations of state, HHL and HHB. For both, we use the volume corrected hadron + Hagedorn gas for $T<165 \mathrm{MeV}$. This is motivated by the fact that the particle ratios using thermal models at RHIC suggest a chemical freeze-out temperature which is close to $165 \mathrm{MeV}$ at the top RHIC energies [28]. Similar results are expected for the top LHC energy also [29].

For the HHL equation of state, we switch over to the lattice equation of state at $T=165 \mathrm{MeV}$. We have found that while the variation of energy density, pressure, and entropy density is rather continuous around the point of switch over Fig,1, a slight discontinuity (of about $3 \%$ ) is seen in the speed of sound. We do not expect it to cause any noticeable difficulty in the final results.

For the HHB, we match our hadronic description to a bag model equation of state, by adjusting the bag pressure to give the transition temperature as $165 \mathrm{MeV}$. The matter at $T>T_{c}$, then consists of non-interacting and mass-less quarks ( $\mathrm{u}, \mathrm{d}$, and $\left.\mathrm{s}\right)$ and gluons, at zero baryonic chemical potential.

In Fig. 3 we have shown the square of the speed of sound for the two equations of state as a function of $\varepsilon^{1 / 4}$, which clearly depicts the vanishing speed of sound for the HHB equation of state, over the energy density covered by the mixed phase.

We reiterate that during the mixed phase, $0.4 \leq \varepsilon \leq 2 \mathrm{GeV} / \mathrm{fm}^{3}$, the speed of sound is zero for the HHB and is larger than that for the HHL equation of state for higher energy densities. The speed of sound for the HHL is never zero. These differences in the speed of sound should lead to interesting differences in the history of evolution of the system, though the integrated effects may still turn out to be small. 

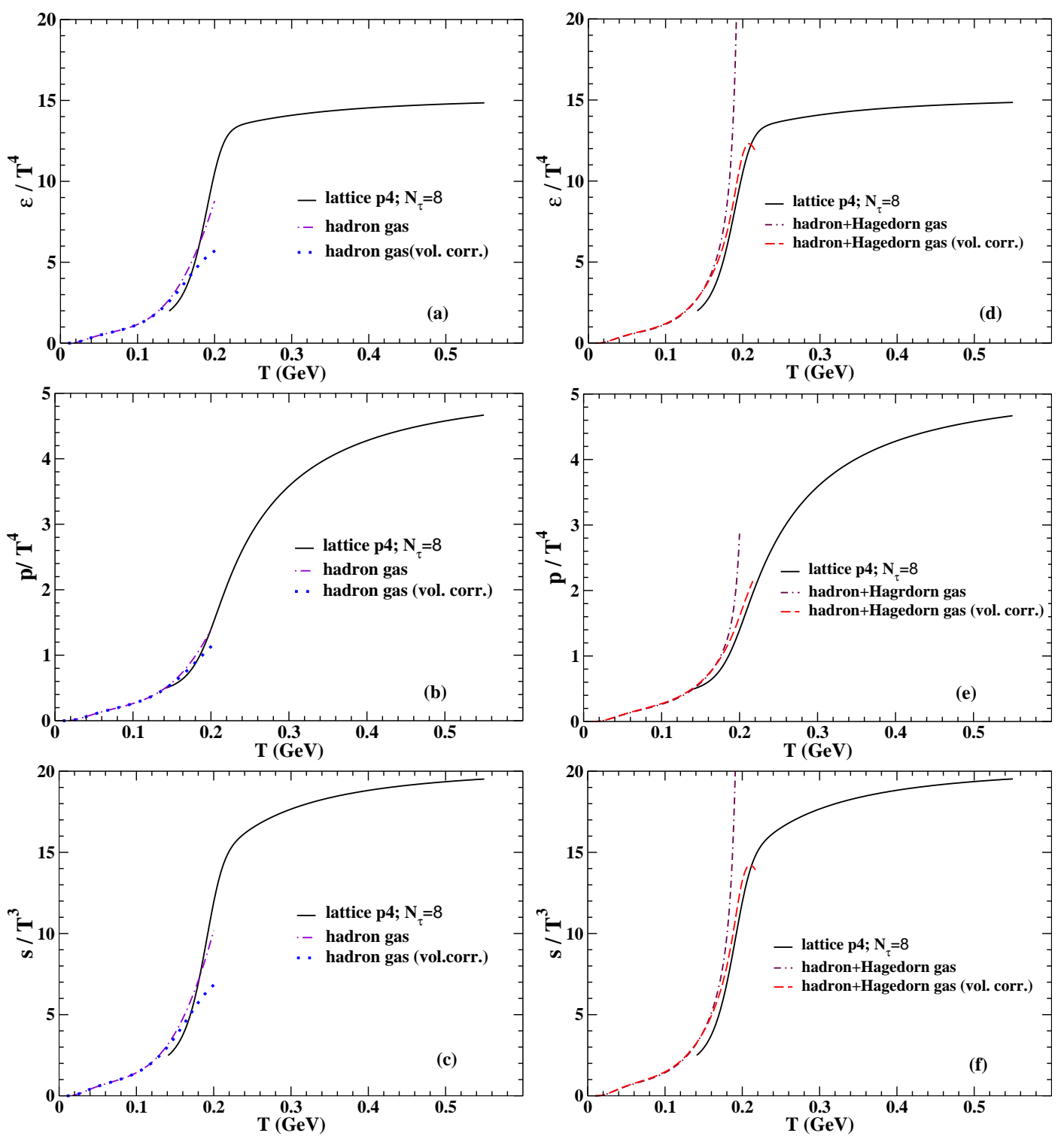

Figure 1. $\varepsilon / T^{4}, p / T^{4}$, and $s / T^{3}$ for hadron gas and volume corrected hadron gas, along with lattice results $(\mathrm{a}, \mathrm{b}$, and $\mathrm{c})$. The panels $\mathrm{d}$, e, and $\mathrm{f}$ depict corresponding results for hadron + Hagedorn gas without and with correction for finite volume of the particles.

\section{Initial Conditions and Particle Spectra}

For $200 \mathrm{~A} \mathrm{GeV} \mathrm{Au+Au} \mathrm{collision} \mathrm{at} \mathrm{RHIC} \mathrm{we} \mathrm{assume} \mathrm{QGP} \mathrm{is} \mathrm{formed} \mathrm{at} \tau_{0}=0.2 \mathrm{fm} / c$ with an energy density used in earlier works [30] which provides a good description to particle spectra and thermal photon spectra obtained experimentally. This is obtained by assuming that a part of the entropy is generated in hard collisions and the remaining part is produced in soft collisions [31] and the relative magnitudes are then adjusted to give the rapidity density of the particles produced in the collisions $d N_{\mathrm{ch}} / d y \approx 680$. At $\mathrm{LHC}(\mathrm{Pb}+\mathrm{Pb} @ 5.5 \mathrm{~A} \mathrm{TeV})$, a smaller value of $\tau_{0}=0.1 \mathrm{fm} / c$ is considered and the 


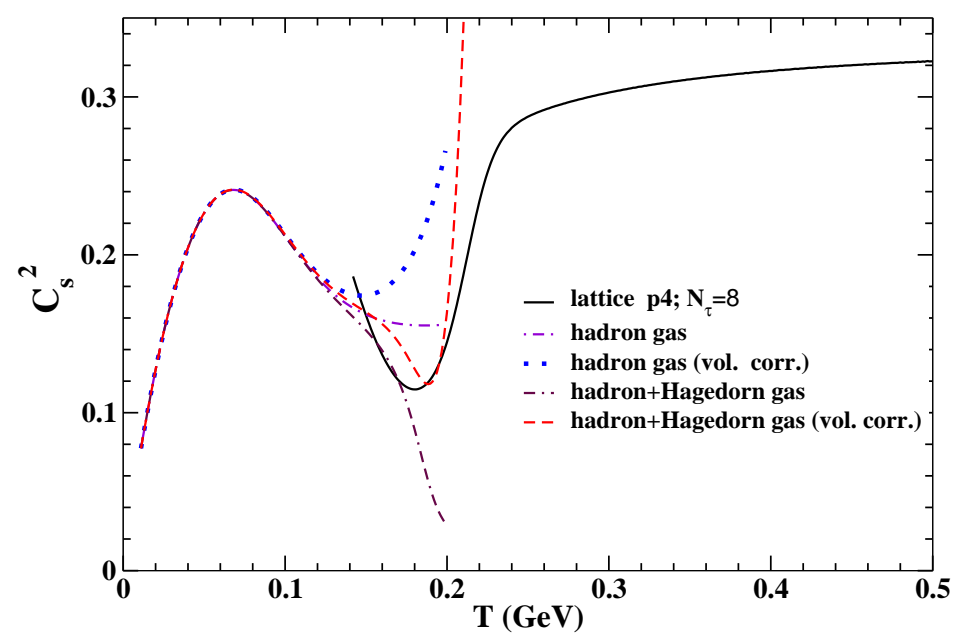

Figure 2. (Colour on-line) Speed of sound for (i) hadron resonance gas, (ii) volume corrected hadron resonance gas, (iii) hadron and Hagedorn resonance gas and (iv) volume corrected hadron and Hagedorn resonance gas with lattice results.

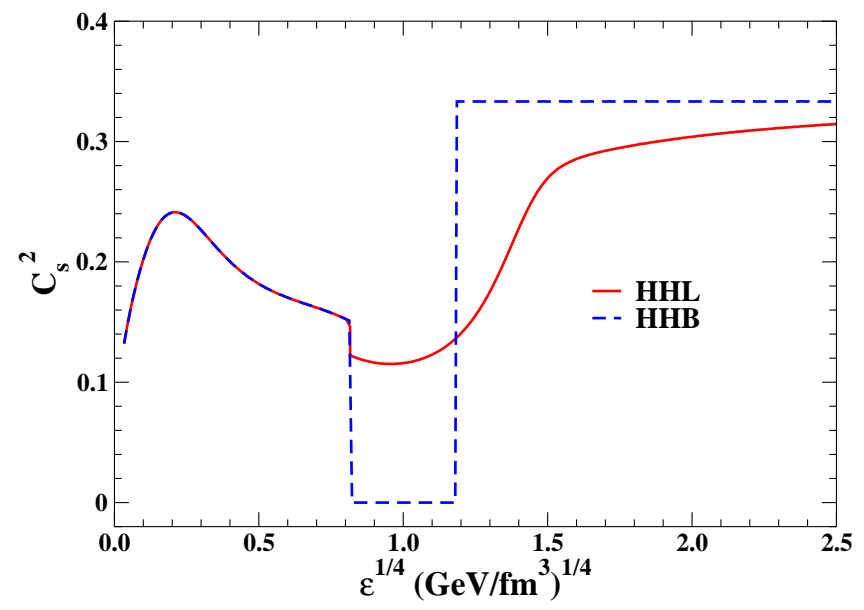

Figure 3. (Colour on-line) Speed of sound for the two equations of state; HHB and HHL.

corresponding rapidity density $d N_{\mathrm{ch}} / d y \approx 2040$ [30].

For both RHIC and LHC, small value of $\tau_{0}$ is considered as we are also interested in thermal photon spectra, which derive a large contribution from early times. If higher formation times are assumed, then the entropy density (and the energy density) can be correspondingly reduced.

The average initial energy densities are:

$$
\left\langle\varepsilon_{0}\right\rangle=\left\{\begin{array}{l}
80.8 \mathrm{GeV} / \mathrm{fm}^{3} \quad \text { at RHIC, } \tau_{0}=0.2 \mathrm{fm} / \mathrm{c}, \\
718.3 \mathrm{GeV} / \mathrm{fm}^{3} \quad \text { at LHC, } \tau_{0}=0.1 \mathrm{fm} / \mathrm{c} .
\end{array}\right.
$$

We numerically solve the hydrodynamics equations [12] for central collisions for a azimuthally symmetric, and longitudinally boost-invariant expansion of the system for 
identical initial conditions and the two equations of state. The freeze-out is assume to take place at $T=100 \mathrm{MeV}$, and the particle spectra are obtained using the Cooper-Frye formula [32. The photon production is calculated over the history of evolution of the system [33]. The rate of production of photons from the quark matter (QM) is taken from the complete leading order calculation of Arnold et al. 34]. The production of photons from the hadronic matter (HM) is estimated using the results from Turbide et al. [35].

For the equation of state incorporating the bag model, the quark fraction during the mixed phase is obtained using a procedure developed earlier [36]. Let us assume that the energy density of the hadronic phase at $T=T_{c}$ is $\varepsilon_{h}\left(T_{c}\right)$ and that for the quarkgluon plasma phase is $\varepsilon_{q}\left(T_{c}\right)$. One can easily show that [36] a fraction $f$ of the energy density $\left(\epsilon\left(f, T_{c}\right)\right)$ in the mixed phase will be contributed by the quark-gluon plasma, where

$$
\varepsilon\left(f, T_{c}\right)=\varepsilon_{q}\left(T_{c}\right) f+\varepsilon_{h}\left(T_{c}\right)(1-f) .
$$

The remaining fraction $(1-f)$ will be contributed by the hadronic matter, as seen above.

For the equation of state incorporating lattice results, we assume that the matter consists of quarks and gluons for $T \geq 185 \mathrm{MeV}$ and below that it is hadronic.

In Fig. 4 the results for transverse momentum distribution of thermal photons, pions, kaons, and protons for central collision of gold nuclei at $y=0$, for the two equations of state, at RHIC energy are shown. The experimental data for $0-5 \%$ most central collisions for the pions, kaons, and protons (from Ref. [37]) are also shown for comparison. No attempts were made to adjust any parameters or normalizations.

Several interesting facts emerge. First of all we note that both the equations of state give a reasonable description to the particle distributions, though a slight preference for the HHL equation of state is easily discernible. Secondly, the inverse slope of the spectra for HHL equation of state is larger than the same for the HHB equation of state, and the largest difference is seen for the spectra for protons.

We feel that the main source of the difference lies in the variation of speed of sound with energy density for the two equations of state. When the system follows HHB equation of state, the acceleration of the expansion is stalled during the mixed phase, which gives rise to a smaller radial velocity at the time of freeze-out. We add that the contribution of resonance decay (which would improve the description of the experimental data at lower $p_{T}$ ) is not included in this analysis.

Similar results have been reported by authors using bag-model and lattice equation of state [40] at RHIC energies. The authors of Ref. [40] also report only a slight difference even for the elliptic flow of pions, though for protons the difference is still noticeable. Their description of the hadronic matter, however, is not as rich as that used in the present work.

We have verified that the thermal photon spectrum is quite close to similar calculations reported earlier [30]. We have also shown the data for single photons for 0- 
20\% most central collisions [38] for a comparison. A complete description of photon data would involve addition of prompt contribution scaled by appropriate nuclear overlapping function [39].

Corresponding results for the top LHC energy are shown in Fig. 5. One can see that the difference between the transverse momentum distribution of photons and the hadrons for the two equations of state is further reduced, though the slight increase in the inverse slope for the HHL equation of state can still be seen. This suggests that the life-time of the system is large enough to greatly obliterate the differences in the momentum distribution of the particles at the time of freeze-out. In fact, for both the cases the difference in the history of evolution which should have affected the thermal photon spectra is also obliterated due to the integration.

These results suggest that the particle spectra (even for thermal photons) can not distinguish between the equations of state, one admitting a first order phase transition, and the other admitting a rapid cross-over as suggested by lattice calculations.

Are we to understand that there is no way to distinguish between these two scenarios? We realise however that the history of evolution of the two systems must be different, as they are subjected to different rates of expansion due to the varying speed of sound.

We recall some early works [41, 42] where it was suggested that a big difference between the so-called $R_{\text {out }}$ and $R_{\text {side }}$ may imply a first order phase transition. The

experimental data at RHIC energies, however point to an $R_{\text {out }} / R_{\text {side }} \approx 1$, which leads to the so-called HBT puzzle [43, 44] at RHIC. It has also spawned several complex questions like what does interferometry of pions actually measure. A definite, though not sufficient, improvement has been reported when a lattice inspired equation of state is used [45. It has also been suggested [45] that several other small corrections add up to fully explain this feature.

We adopt a two-pronged approach in the following. First, we trace the history of evolution of the systems, both at RHIC and LHC, for the two equations of state.

Next, we study the intensity interferometry of thermal photons, which should be free from such complications and which should be sensitive to the history of the evolution of the system. We shall see that this could be quite rewarding indeed.

\section{History of Evolution of the Collision}

The energy-density weighted average temporal evolution of energy density, temperature, and the radial velocity for central collisions at RHIC and LHC energy, are shown in Fig. 6 following the two equations of state, HHB and HHL.

The average is obtained as:

$$
\langle f\rangle=\frac{\int 2 \pi r d r f(r, \tau) \varepsilon(r, \tau)}{\int 2 \pi r d r \varepsilon(r, \tau)} .
$$

We see (Fig. 6) that both at RHIC and LHC, the variation of the energy density with time for the two equations of state is quite similar, though the numerical values 

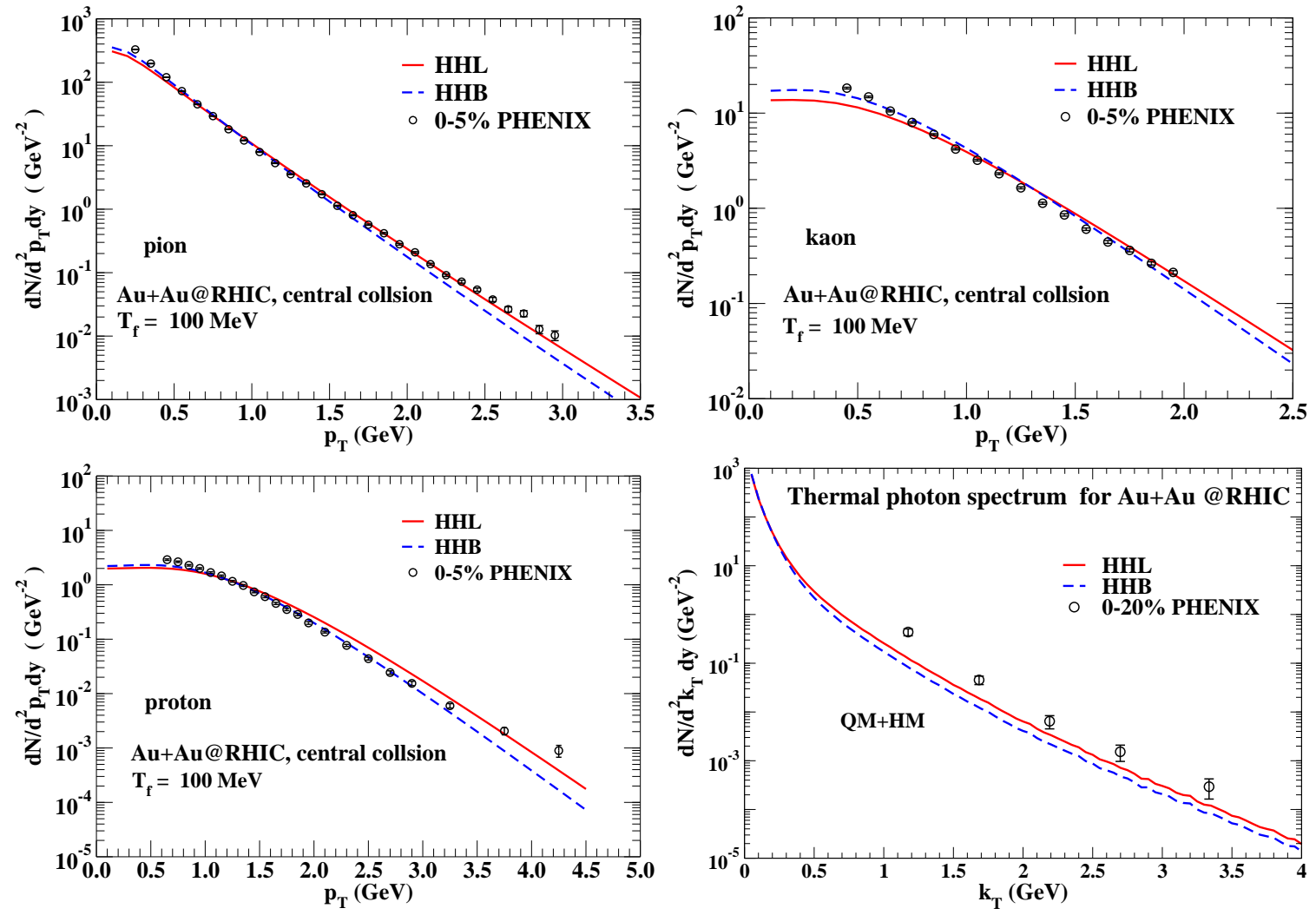

Figure 4. (Colour on-line) Pion, kaon, proton and thermal photon $p_{T}$ spectra at RHIC for the equations of state, HHB and HHL. All the calculations are for impact parameter $\mathrm{b}=0 \mathrm{fm}$. The experimental data for hadrons ( $0-5 \%$ centrality bin) are taken from [37] and photon data for 0-20\% centrality bin from [38] (see text for detail).

show a marginally rapid decrease in $\langle\varepsilon\rangle$ at early times for the HHB. This is due to a larger value of speed of sound in the QGP phase for the HHB equation of state (see. Fig. 3).

The time variation of the temperature is a little more interesting as it stays nearly constant when bulk of the system is in the mixed phase for the HHB equation of state, whereas it continues to decrease for the other case.

The most interesting and potentially useful variation is observed for the radial velocity for the two equations of state. The HHB equation of state gives rise to a larger $v_{T}$ during the early QGP phase. The rise of the radial velocity stalls, once the system enters a mixed phase and then rises again. The radial velocity for the HHL equation of state rises continuously, stays below that for the HHB equation of state, but overshoots it once the former stalls due to the onset of the mixed phase. The final $v_{T}$ for the HHL EOS is slightly larger than that for the HHB. We have already seen the consequence of this in the particle spectra (Figs. 4 and [5). 

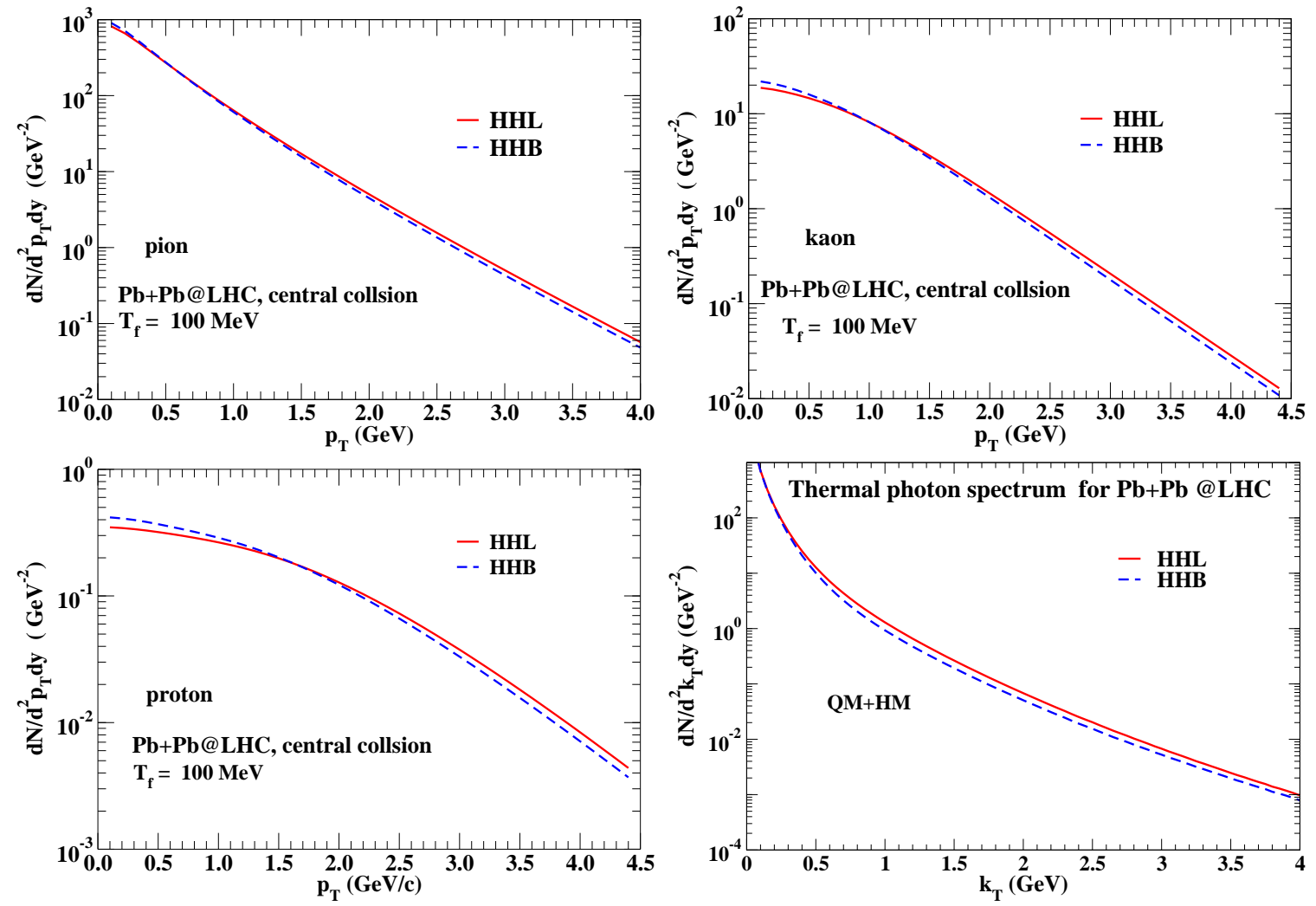

Figure 5. (Colour on-line) Same as Fig. 4 at LHC.

\section{Intensity Interferometry of Thermal Photons}

The intensity interferometry of thermal photons has been suggested as a valuable probe for the history of evolution of the system formed in relativistic heavy ion collisions [46, 47, 48]. Two recent studies (Ref. [49] and Ref. [50]) have further refined these early expectations. The authors of Ref. [49] report an exciting possibility of observation of interference of photons from the quark-phase and the hadronic phase, while Ref. [50] explores the details of angle-dependent intensity correlation of thermal photons for non-central collisions.

The WA98 collaboration [51] has already succeeded in such a study at SPS energy with interesting results. It is expected that the increased initial temperature, additional sources of photons [52, 53, 54], and jet-quenching (which suppresses the production of $\pi^{0}$ at larger $p_{T}$ ) may make this study more feasible at RHIC and LHC energies.

The correlation function between two photons with momenta $k_{1}$ and $k_{2}$ is defined in general as:

$$
C\left(\mathbf{k}_{\mathbf{1}}, \mathbf{k}_{\mathbf{2}}\right)=\left[E_{1} E_{2} \frac{d N}{d^{3} k_{1} d^{3} k_{2}}\right] /\left[E_{1} \frac{d N}{d^{3} k_{1}} E_{2} \frac{d N}{d^{3} k_{2}}\right],
$$

where the numerator gives the correlated spectrum and the denominator denotes the product of single spectrum of the two photons. This can be written in terms of the 

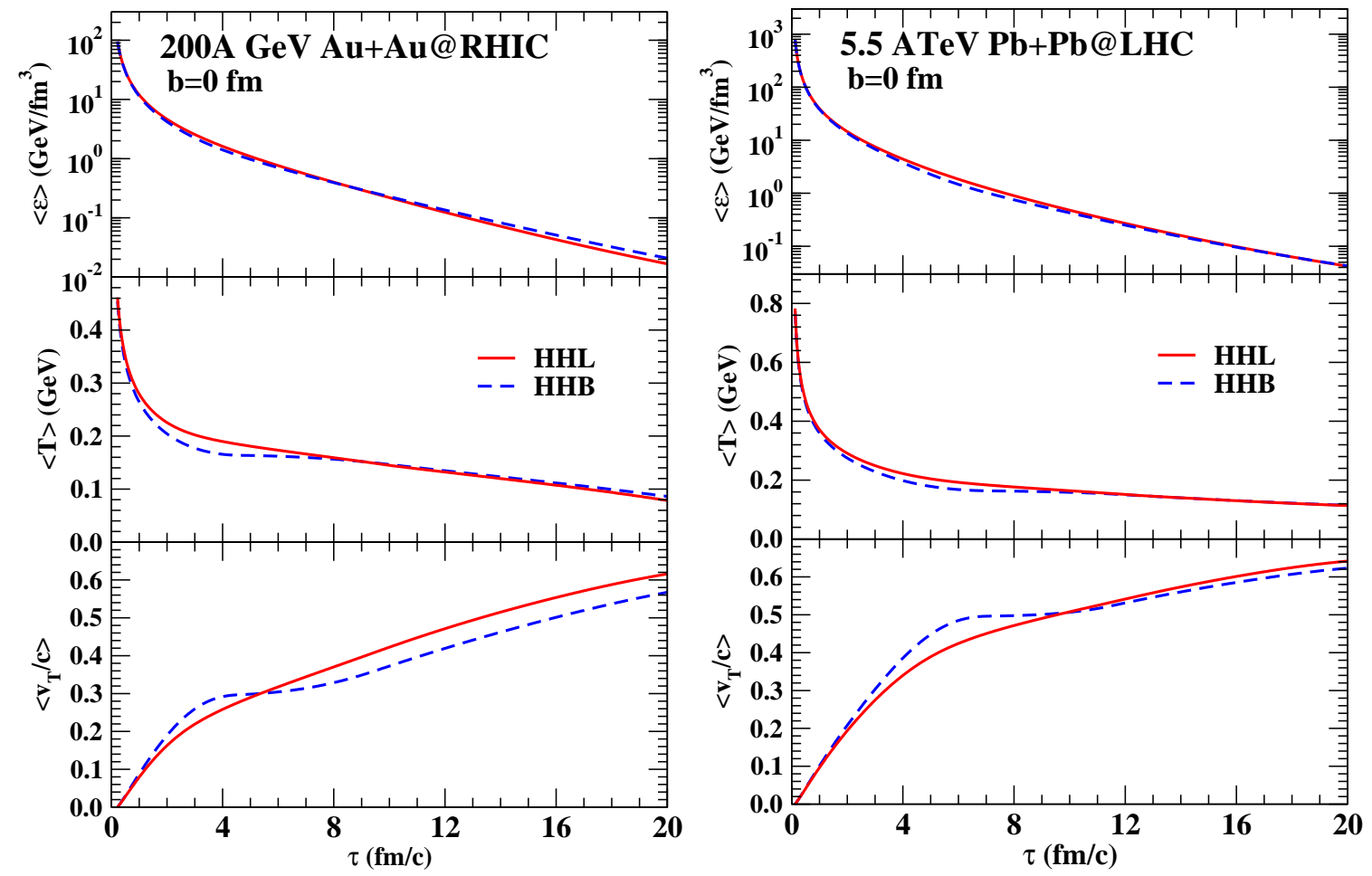

Figure 6. (Colour on-line) Temporal evolution of average energy density, temperature, and radial flow velocity at RHIC (left panel) and LHC (right panel) for the HHB and HHL equations of state.

source-function as

$$
C(\mathbf{q}, \mathbf{K})=1+\frac{1}{2} \frac{\left|\int d^{4} x S(x, \mathbf{K}) e^{i x \cdot q}\right|^{2}}{\int d^{4} x S\left(x, \mathbf{k}_{\mathbf{1}}\right) \int d^{4} x S\left(x, \mathbf{k}_{\mathbf{2}}\right)} .
$$

In the above, $S(x, \mathbf{K})$ is the space-time emission function, and

$$
\mathbf{q}=\mathbf{k}_{\mathbf{1}}-\mathbf{k}_{\mathbf{2}}, \mathbf{K}=\left(\mathbf{k}_{\mathbf{1}}+\mathbf{k}_{\mathbf{2}}\right) / 2 .
$$

The factor ' $1 / 2$ ' in Eq. (14) arises from the averaging over the spins of the photons in the final state. The space-time emission function $S$ is approximated as the photon production rate, $E d N / d^{4} x d^{3} k$, from the quark and the hadronic matter phases. The source function $S$ is expressed as $S_{Q}+S_{H}$ in the numerator, where $Q$ and $H$ stand for quark and hadronic phases respectively. The contributions of the quark matter or the hadronic matter or both to the correlation of photons are estimated by retaining $S_{Q}$ or $S_{H}$ or both.

The correlation function $C(\mathbf{q}, \mathbf{K})$ is often represented in terms of the outward, side-ward, and longitudinal momentum differences, $q_{o}, q_{s}$ and $q_{\ell}$. We can write the 4-momentum $\left(k_{i}^{\mu}\right)$ of the $i$ th photon as:

$$
k_{i}^{\mu}=\left(k_{i T} \cosh y_{i}, k_{i T} \cos \psi_{i}, k_{i T} \sin \psi_{i}, k_{i T} \sinh y_{i}\right)
$$

where $k_{T}$ is the transverse momentum, $y$ is the rapidity, and $\psi$ is the azimuthal angle. 
The difference of transverse momenta is defined as

$$
\mathbf{q}_{\mathbf{T}}=\mathbf{k}_{\mathbf{1 T}}-\mathbf{k}_{\mathbf{2}} \mathbf{T}
$$

and the average transverse momentum

$$
\mathbf{K}_{\mathbf{T}}=\left(\mathbf{k}_{\mathbf{1 T}}+\mathbf{k}_{\mathbf{2}} \mathbf{T}\right) / 2 .
$$

In terms of these variables, the longitudinal, outward, and side-ward momentum differences are obtained as [46]:

$$
\begin{aligned}
q_{\ell} & =k_{1 z}-k_{2 z} \\
& =k_{1 T} \sinh y_{1}-k_{2 T} \sinh y_{2}, \\
q_{o} & =\frac{\mathbf{q}_{\mathbf{T}} \cdot \mathbf{K}_{\mathbf{T}}}{K_{T}} \\
& =\frac{\left(k_{1 T}^{2}-k_{2 T}^{2}\right)}{\sqrt{k_{1 T}^{2}+k_{2 T}^{2}+2 k_{1 T} k_{2 T} \cos \left(\psi_{1}-\psi_{2}\right)}}, \\
q_{s} & =\left|\mathbf{q}_{\mathbf{T}}-q_{o} \frac{\mathbf{K}_{\mathbf{T}}}{K_{T}}\right| \\
& =\frac{2 k_{1 T} k_{2 T} \sqrt{1-\cos ^{2}\left(\psi_{1}-\psi_{2}\right)}}{\sqrt{k_{1 T}^{2}+k_{2 T}^{2}+2 k_{1 T} k_{2 T} \cos \left(\psi_{1}-\psi_{2}\right)}} .
\end{aligned}
$$

The radii corresponding to these momentum differences are obtained by approximating the correlation function as,

$$
C\left(q_{o}, q_{s}, q_{\ell}\right)=1+\frac{1}{2} \exp \left[-\left(q_{o}^{2} R_{o}^{2}+q_{s}^{2} R_{s}^{2}+q_{\ell}^{2} R_{\ell}^{2}\right)\right] .
$$

The root mean square momentum difference $\left\langle q_{i}^{2}\right\rangle$ is,

$$
\left\langle q_{i}^{2}\right\rangle=\frac{\int(C-1) q_{i}^{2} d q_{i}}{\int(C-1) d q_{i}},
$$

and for the Gaussian parameterization shown above, this becomes

$$
R_{i}^{2}=\frac{1}{2\left\langle q_{i}^{2}\right\rangle}
$$

As a first step we plot the spatial and the temporal distributions of the sources obtained for the two equations of state in Fig. 7, for a typical momentum of the photons $K_{T} \approx 1.7 \mathrm{GeV}$, which provide valuable information about the dynamics of the system.

We find that the HHL equation of state leads to a larger production of photons at smaller radial distances as well at intermediate times. The spectrum of the photons in Fig. 4, already indicates this. The photons from quark matter originate at early times and those from hadronic matter at later times, and as the interference between the two depends on their relative contributions [49], this difference holds out a hope that we may see a difference in the intensity interferometry of photons for the two equations of state. 

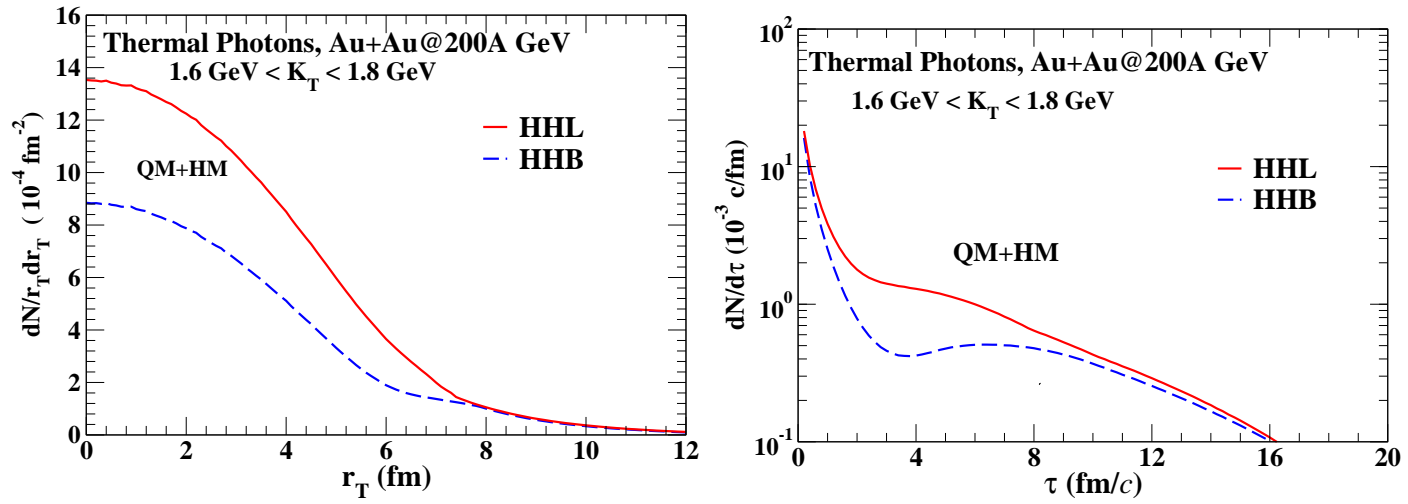

Figure 7. (Colour on-line) The radial (left panel) and temporal dependence (right panel) of the source of photons for HHL and HHB equations of state for central collision of gold nuclei at the top RHIC energy.
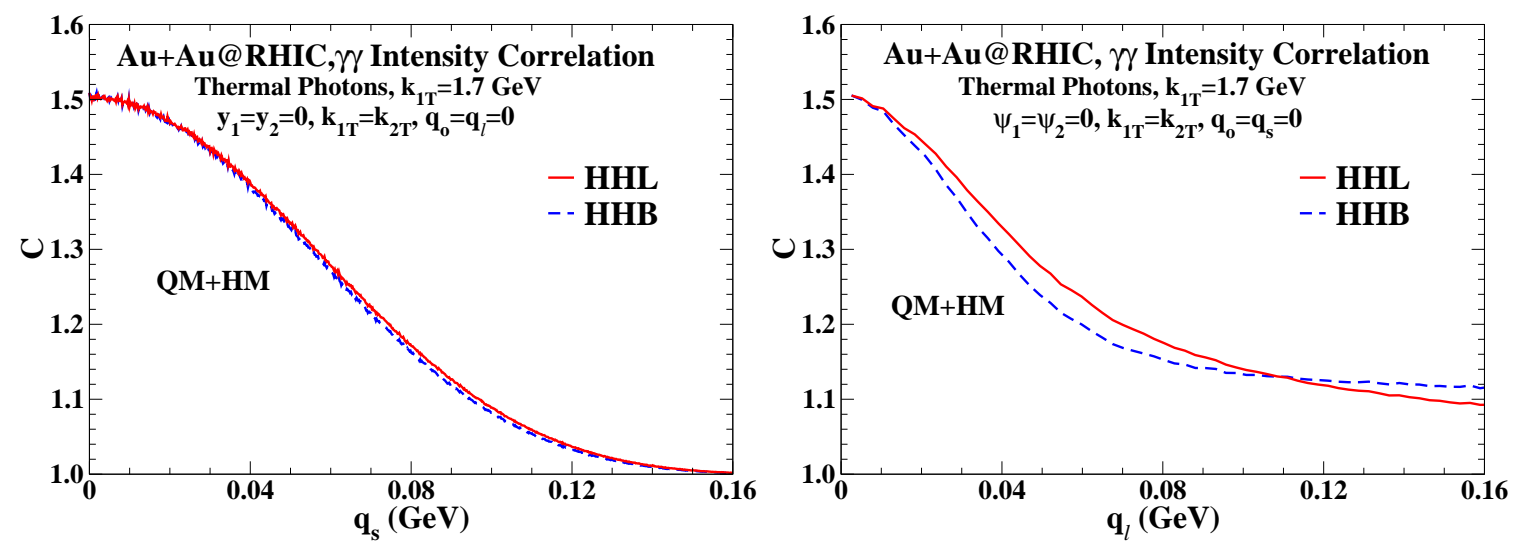

Figure 8. (Colour on-line) The sideward (left panel) and longitudinal correlation (right panel) functions for thermal photons produced in central collision of gold nuclei at RHIC.

In order to bring out the dependence of the correlation function on the outward, sideward, and longitudinal momentum differences very clearly, we choose momenta of the interfering photons such that when $q_{o} \neq 0$, then $q_{s}$ and $q_{l}$ are zero, and so on.

The corresponding side-ward and longitudinal correlation functions are shown in Fig. 8. We see that while the sideward correlation is essentially identical for the two equations of state, the longitudinal correlation function shows a mild variation when we change the equation of state.

The results for the outward correlation (Fig. 9) are more dramatic and along with the interference already reported in Ref. [49, we see a very clear difference in the results obtained for the equations of state. Recalling that this interference is decided by the relative contribution of the hadronic and quark phase, we also show the quark matter and hadronic matter contributions to the correlation function. We notice a clear difference in the underlying radii for the hadronic matter contribution for the equations of state; the one for the HHB equation of state being effectively much larger. 

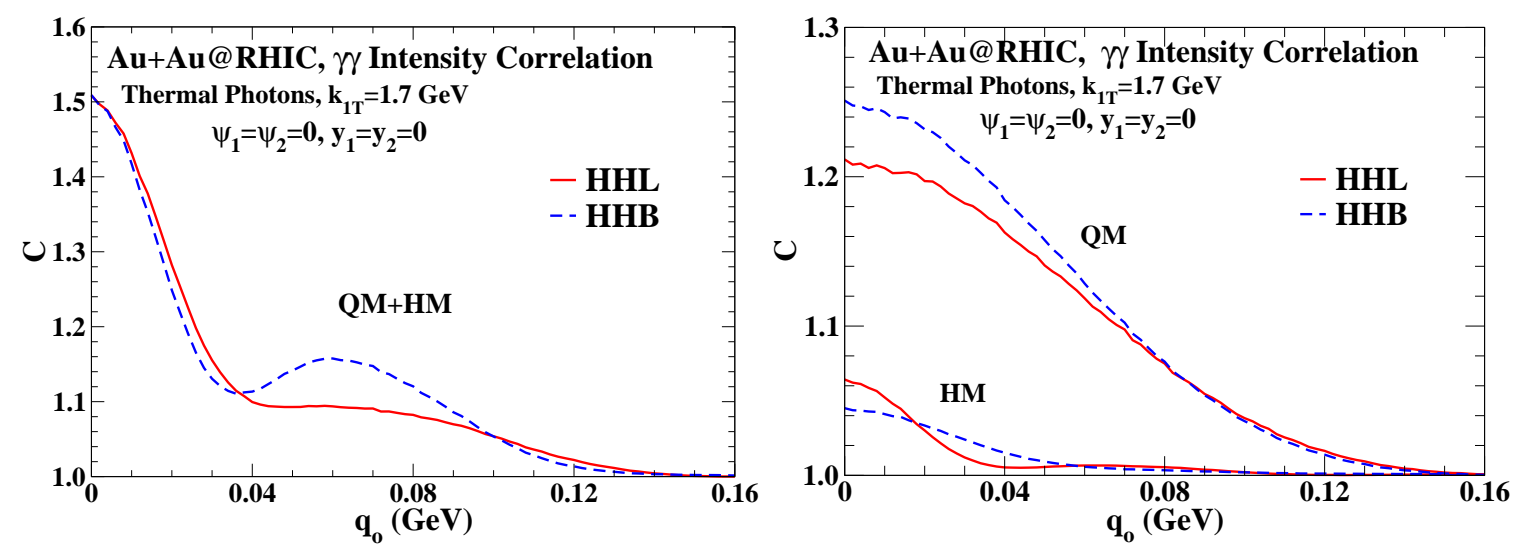

Figure 9. (Colour on-line) Left panel: The outward correlation function for thermal photons produced in central collision of gold nuclei at RHIC. Right panel: Individual contributions of quark matter and hadronic matter to the correlation.

We note that in general the experimentally measured correlation functions (see, e.g., Ref. [51]) have larger error-bars for smaller values of the momentum differences. However, these measurement normally have smaller error-bars for larger values of momentum differences, where our results are seen to be most sensitive to the differences in the equations of state. This holds out the hope that these differences can be experimentally verified.

We follow the treatment of Ref. [49] to estimate the source-sizes of the HM and QM contributions along with their separation (see later) and write:

$$
C\left(q_{i}, \alpha\right)=1+0.5\left|\rho_{i, \alpha}\right|^{2}
$$

where $i=o, s$, or $\ell$, and $\alpha$ denotes quark matter (Q) and hadronic matter $(\mathrm{H})$ in an obvious notation.

Next we approximate the source distributions $\left|\rho_{i, \alpha}\right|$ as a Gaussian and write:

$$
\left|\rho_{i, \alpha}\right|=I_{\alpha} \exp \left[-0.5\left(q_{i}^{2} R_{i, \alpha}^{2}\right)\right]
$$

where $I_{Q}=d N_{Q} /\left(d N_{Q}+d N_{H}\right)$ and $I_{H}=d N_{H} /\left(d N_{Q}+d N_{H}\right)$. The final correlation functions are then approximated as,

$$
C\left(q_{i}\right)=1+0.5\left[\left|\rho_{i, Q}\right|^{2}+\left|\rho_{i, H}\right|^{2}+2\left|\rho_{i, Q}\right|\left|\rho_{i, H}\right| \cos \left(q_{i} \Delta R_{i}\right)\right]
$$

which clearly reveals the interference between the two sources. Here $\Delta R_{i}$ stands for the separation of the two sources. Thus for example, for thermal photons having $K_{T} \approx 1.7$ $\mathrm{GeV}$ at $\mathrm{RHIC}$, the radii (in $\mathrm{fm}$ ) corresponding to each source are obtained as:

HHB

$$
R_{o, Q}=2.5, R_{o, H}=8.3, \Delta R_{o}=14.9 .
$$

HHL

$$
R_{o, Q}=2.7, R_{o, H}=4.8, \Delta R_{o}=13.4 .
$$



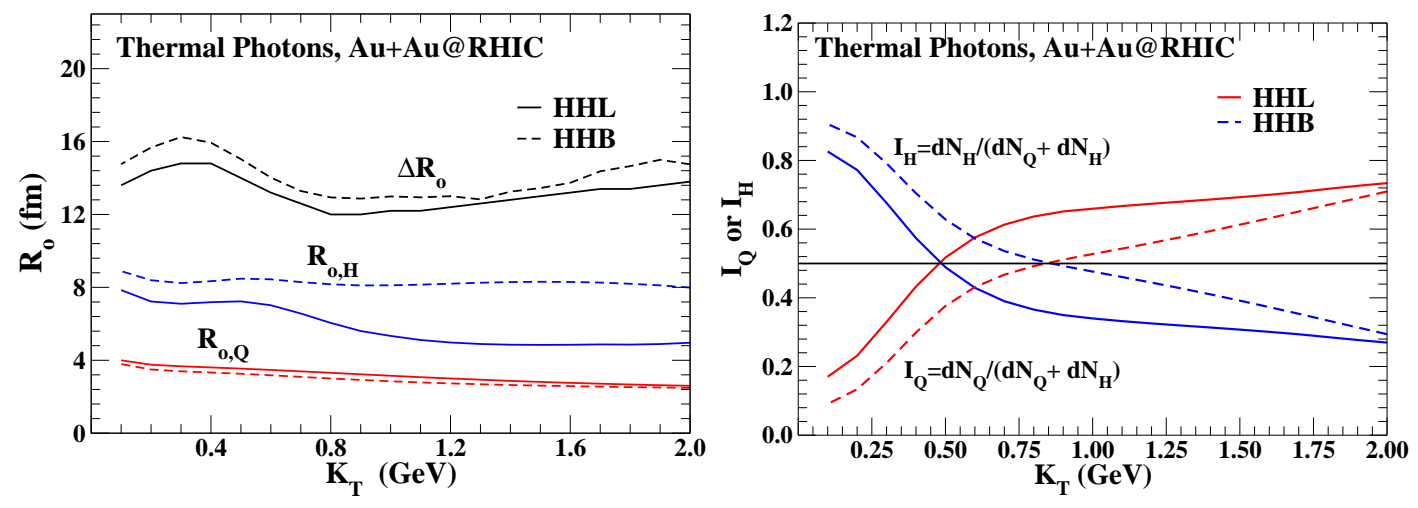

Figure 10. (Colour on-line) Left panel: Transverse momentum dependence of outward radii for hadronic and quark matter sources for thermal photons. Right panel: The fraction of thermal photons emitted from quark matter and hadronic matter.

The transverse momentum dependence of the radii and the separation $\Delta R$ for the outward correlation function is shown in the left panel of Fig. 10. We see that the separation $\Delta R_{o}$, which corresponds to the life time of the system is slightly smaller for the HHL equation, as it does not incorporate a mixed phase (see discussion in Ref. [49]). One can obtain similar results for the contributions for the sideward and longitudinal radii. The $R_{o}$ for the hadronic matter for the HHL equation of state is also much smaller for the same reason.

The transverse momentum dependence of the quark and the hadronic matter fractions $I_{Q}$ and $I_{H}$ are shown in the right panel of Fig. 10 where, $I_{Q}$ for HHL is found to be larger than that for HHB. As the speed of sound for the HHL equation of state is smaller during the QGP phase (see Fig. 3), the system cools at a slower rate, which leads to a larger contribution from the quark phase. We also find that for HHL equation of state, the quark and hadronic matter contributions become equal $\left(I_{Q}=I_{H}=0.5\right)$ at a slightly lower $K_{T}$.

Next we consider correlation of thermal photons from central collision of lead nuclei at LHC.

The results for the sideward and the longitudinal correlations for transverse momentum of about $1.2 \mathrm{GeV}$ are shown in Fig. 11. It is observed that, while the sideward correlation is again nearly identical for the two equations of state, the longitudinal correlation is fairly different and quite sensitive to the equation of state used. In view of the importance of this observation it would be interesting to analyze this behaviour using a $3+1 \mathrm{D}$ hydrodynamics [55].

The results for the outward correlation function and its composition in terms of contributions from the quark matter and the hadronic matter are shown in Fig. 12. Once again a very distinctive sensitivity is observed for the two equations of state. 

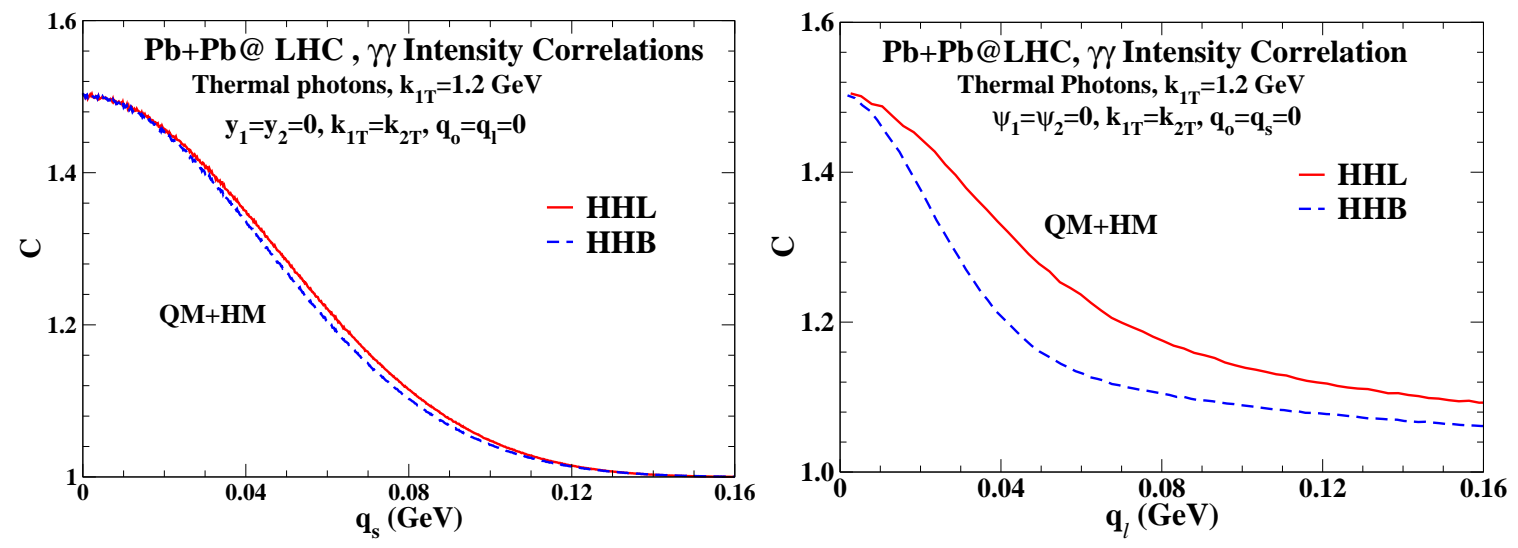

Figure 11. (Colour on-line) The sideward (left panel) and longitudinal correlation (right panel) functions for thermal photons produced in central collision of lead nuclei at LHC.
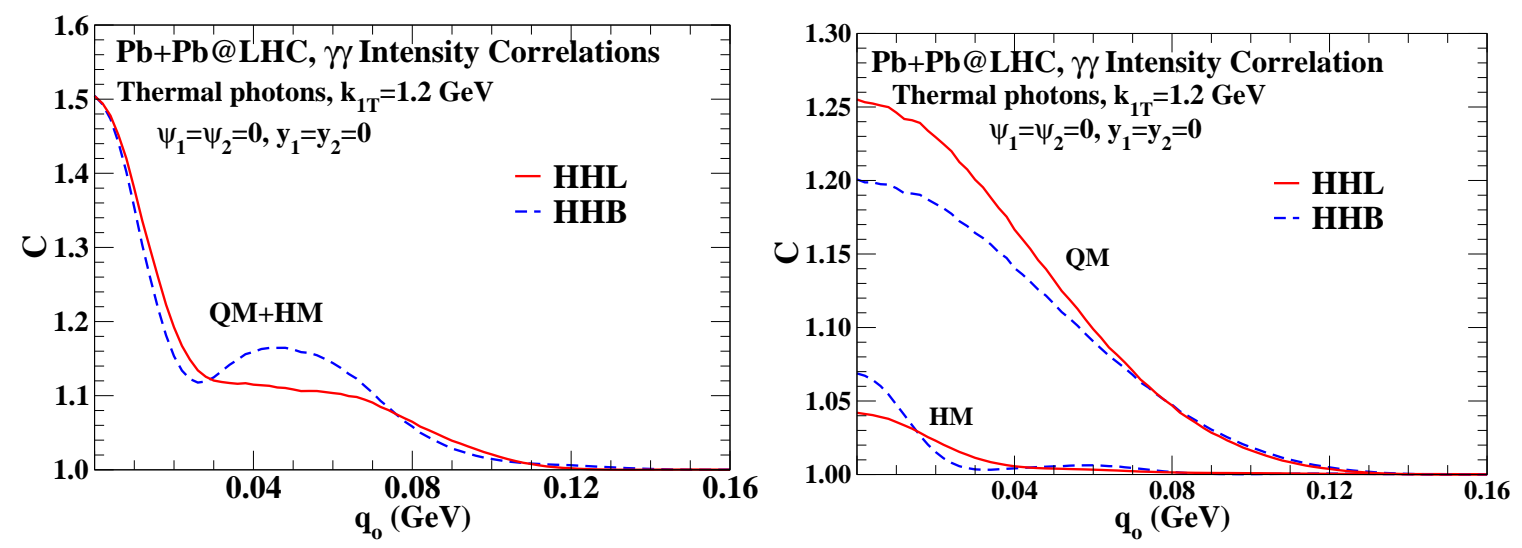

Figure 12. (Colour on-line) The outward correlation functions for thermal photons produced in central collision of lead nuclei at LHC for equation of state HHB and HHL.

\section{Summary and Discussions}

In brief, we have constructed a rich description of hadronic matter at zero baryonic chemical potential by including all the mesons having $M<1.5 \mathrm{GeV}$, and all the baryons having $M<2 \mathrm{GeV}$, in thermal and chemical equilibrium along with Hagedorn resonances. Incorporation of finite volume for the hadrons gives results, which join smoothly with those with the recent lattice calculations. We construct two equations of state by switching over to a bag model equation of state (leading to a first order phase transition) or to the lattice equation of state (showing a rapid crossover around $185 \mathrm{MeV}$ ) at a temperature of $165 \mathrm{MeV}$.

We find that spectra of pions, kaons, protons and thermal photons both at RHIC and LHC energies are quite similar for a given initial condition. However, the experimental data at RHIC show a slight preference for the equation of state incorporating lattice results. 
Still, the longitudinal correlation to a lesser extent and the outward correlation to a great extent, for thermal photons are found to be sensitive to the equation of state. If verified in experiments, this could prove to be a very valuable probe for the equation of state of strongly interacting matter. We have explained these observations by having a close look at the history of evolution of the systems.

Before closing, we note that these studies could be improved in some ways. One could, for example, investigate the consequences of inclusion of viscous effects and using a 3+1D dimensional hydrodynamics, especially for the longitudinal correlation. This will also free our findings from the constraint of the longitudinal boost-invariance assumed here. It would also be of interest to extend this study to non-central collisions, which we shall do shortly. Consequences of varying some of the initial conditions, e.g., formation time and colour gluon condensate inspired initial conditions could also be studied. It may also be worth-while to vary the transition temperature for the two equations of state and study its consequences.

Future directions would involve extending these studies to non-zero baryonic chemical potential, especially in view of the low energy runs at RHIC and upcoming studies at FAIR, though we await more accurate lattice results for non-zero $\mu_{B}$.

In a forthcoming publication we shall extend the present work to study the elliptic flow of dileptons as well as the elliptic flow of photons [56].

\section{Acknowledgements}

Two of us, SD and RC are grateful to Department of Atomic Energy for financial support during the course of this work. We thank M. Cheng, S. Dutta, and S. Gupta for useful correspondence.

\section{References}

[1] I. Arsene et al. [BRAHMS Collaboration], Nucl. Phys. A 757, 1 (2005); K. Adcox et al [PHENIX Collaboration], Nucl. Phys. A 757, 184 (2005); B. B. Back et al. [PHOBOS Collaboration], Nucl. Phys. A 757, 28 (2005); J. J. Adams et al. [STAR Collaboration], Nucl. Phys. A 757, $102(2005)$.

[2] R. A. Lacey et al., Phys. Rev. Lett. 98, 092301 (2007).

[3] X. N. Wang, Phys. Rev. C 63, 054902 (2001); M. Gyulassy, I. Vitev, and X. N. Wang, Phys. Rev. Lett. 86, 2537 (2001).

[4] K. Adcox et al. [PHENIX Collaboration], Phys. Rev. Lett. 88, 022301 (2002); J. Adams et al. [STAR Collaboration], Phys. Rev. Lett. 91, 172302 (2003).

[5] C. Adler et al. [STAR Collaboration], Phys. Rev. Lett. 87, 182301 (2001); ibid 89, 132301 (2002); ibid 90, 032301 (2003); S. S. Adler et al. [PHENIX Collaboration], Phys. Rev. Lett. 91, 182301 (2003).

[6] P. Huovinen, P. F. Kolb, U. Heinz, P. V. Ruuskanen, and S. A. Voloshin, Phys. Lett. B503, 58 (2001); D. Teaney, J. Lauret, and E. V. Shuryak, nucl-th/0110037.

[7] V. Greco, C. M. Ko, and P. Levai, Phys. Rev. Lett. 90, 202302 (2003); R. J. Fries, B. Müller, C. Nonaka, and S. A. Bass, Phys. Rev. Lett. 90, 202303 (2003).

[8] A. Adare et al. [PHENIX Collaboration], Phys. Rev. Lett. 104, 132301 (2010) D. G. d'Enterria and D. Peressounko, Eur. Phys. J. C 46, 451 (2006). 
[9] See e.g., K. Geiger and B. Muller, Nucl. Phys. B 369, 600 (1992); S. A. Bass, B. Muller, and D. K. Srivastava, Phys. Lett. B 551, 277 (2003); Z. Xu and C. Greiner, Phys. Rev. C 71, 064901 (2005).

[10] S. A. Bass and A. Dumitru, Phys. Rev. C 61, 064909 (2000).

[11] See e.g., J. Bjoraker and R. Venugopalan, Phys. Rev. C 63, 024609 (2001).

[12] H. Von Gersdorff, L. D. McLerran, M. Kataja and P. V. Ruuskanen, Phys. Rev. D 34, 794 (1986); J. Cleymans, K. Redlich, and D. K. Srivastava, Phys. Rev. C 55, 1431 (1997).

[13] T. S. Biro, E. van Doorn, B. Muller, M. H. Thoma, and X. N. Wang, Phys. Rev. C 48, 1275 (1993).

[14] K. J. Eskola, B. Muller, and X. N. Wang, Phys. Lett. B 374, 20 (1996).

[15] D. K. Srivastava, M. G. Mustafa, and B. Muller, Phys. Rev. C 56, 1064 (1997).

[16] M. Asakawa, S. A. Bass, and B. Muller, Phys. Rev. Lett. 96, 252301 (2006), P. Romatschke and U. Romatschke, Phys. Rev. Lett. 99, 172301 (2007); H. Song and U. W. Heinz, J. Phys. G 36, 064033 (2009); N. Demir and S. A. Bass, Phys. Rev. Lett. 102, 172302 (2009); A. K. Chaudhuri, J. Phys. G 37, 075011 (2010).

[17] A. Bazavov et al., Phys. Rev. D 80, 014504 (2009).

[18] G. M. Welke, R. Venugopalan, and M. Prakash, Phys. Lett. B 245, 2 (1990).

[19] R. Hagedorn, Nuovo Cim. Suppl. 3, 147 (1965).

[20] J. Noronha-Hostler, M. Beitel, C. Greiner, and I. Shovkovy, Phys. Rev. C 81, 054909 (2010).

[21] S. Chatterjee, R. M. Godbole, and S. Gupta, Phys. Rev. C 81, 044907 (2010); M. Cheng, J. Phys. Conf. Ser. 230, 012015 (2010).

[22] A. Majumder and B. Muller, arXiv:1008.1747 [hep-ph].

[23] M. Panero, Phys. Rev. Lett. 103, 232001 (2009).

[24] J. Cleymans, K. Redlich, H. Satz, and E. Suhonen, Z. Phys. C 33, 151 (1986).

[25] See e.g., J. Sollfrank, P. Huovinen, M. Kataja, P. V. Ruuskanen, M. Prakash, and R. Venugopalan, Phys. Rev. C 55, 392 (1997).

[26] R. Hagedorn and J. Rafelski, Phys. Lett. B 97, 136 (1980).

[27] J. I. Kapusta and K. A. Olive, Nucl. Phys. A 408, 478 (1983).

[28] A. Andronic, P. Braun-Munzinger and J. Stachel Phys. Lett. B 673142 (2009); J. Cleymans, H. Oeschler, K. Redlich, and S. Wheaton Phys. Rev. C 73, 034905 (2006).

[29] I. Kraus, J. Cleymans, H. Oeschler, K. Redlich, and S. Wheaton, arXiv:0902.0873 [hep-ph].

[30] E. Frodermann, R. Chatterjee and U. Heinz, J. Phys. G 34, 2249 (2007), and references there-in.

[31] P. F. Kolb and U. W. Heinz, "Hydrodynamic description of ultrarelativistic heavy-ion collisions," arXiv:nucl-th/0305084.

[32] F. Cooper and G. Frye, Phys. Rev. D 10, 186 (1974).

[33] See, e.g, D. K. Srivastava and B. Sinha, Phys. Rev. C 64, 034902 (2001); R. Chatterjee, D. K. Srivastava, and S. Jeon, Phys. Rev C 79034906 (2009), and references there in.

[34] P. Arnold, G. D. Moore, and L. G. Yaffe, JHEP 12, 009 (2001).

[35] S. Turbide, R. Rapp, and C. Gale, Phys. Rev. C 69, 014903 (2004).

[36] K. Kajantie, J. I. Kapusta, L. D. McLerran, and A. Mekjian, Phys. Rev. D 34, 2746 (1986).

[37] S. S. Adler et al. [PHENIX Collaboration], Phys. Rev. C 69, 034909 (2004).

[38] A. Adare et al., Phys. Rev. Lett. 104, 132301 (2010).

[39] P. Aurenche, M. Fontannaz, J.Ph. Guillet, B. A. Kniehl, E. Pilon, and M. Werlen, Eur. Phys. J. C 9, 107 (1999); P. Aurenche, M. Fontannaz, J.Ph. Guillet, E. Pilon, and M. Werlen, Phys. Rev. D 73, 094007 (2006).

[40] P. Huovinen, Nucl. Phys. A 761, 296 (2005); P. Huovinen and P. Petreczky, Nucl. Phys. A 837, 26 (2010).

[41] S. Pratt, Phys. Rev. D 33, 1314 (1986); G. Bertsch, M. Gong and M. Tohyama, Phys. Rev. C 37, 1896 (1988); G. F. Bertsch, Nucl. Phys. A 498, 173C (1989).

[42] D. H. Rischke and M. Gyulassy, Nucl. Phys. A 608, 479 (1996).

[43] S. Soff, S. A. Bass and A. Dumitru, Phys. Rev. Lett. 86, 3981 (2001) 
[44] U. W. Heinz and P. F. Kolb, hep-ph/0204061; U. W. Heinz and P. F. Kolb, Nucl. Phys. A 702, 269 (2002).

[45] S. Pratt, Acta Phys. Polon. B 40, 1249 (2009).

[46] D. K. Srivastava, Phys. Rev. C 71, 034905 (2005).

[47] D. K. Srivastava and J. I. Kapusta, Phys. Lett. B 307, 1 (1993); D. K. Srivastava and J. I. Kapusta, Phys. Rev. C 48, 1335 (1993); D. K. Srivastava, Phys. Rev. D 49, 4523 (1994); D. K. Srivastava and C. Gale, Phys. Lett. B319, 407 (1993); D. K. Srivastava and J. I. Kapusta, Phys. Rev. C 50, 505 (1994); S. A. Bass, B. Müller, and D. K. Srivastava, Phys. Rev. Lett. 93, 162301 (2004).

[48] A. Timmermann, M. Plümer, L. Razumov, and R. M. Weiner, Phys. Rev. C 50, 3060 (1994); J. Pisut, N. Pisutova, and B. Tomasik, Phys. Lett. B 345, 553 (1995), [Erratum-ibid. B 353, 606 (1995)]; C. Slotta and U. W. Heinz, Phys. Lett. B 391, 469 (1997); D. Peressounko, Phys. Rev. C 67, 014905 (2003); J. Alam, B. Mohanty, P. Roy, S. Sarkar, and B. Sinha, Phys. Rev. C 67, 054902 (2003); T. Renk, hep-ph/0408218.

[49] D. K. Srivastava and R. Chatterjee, Phys. Rev. C 80, 054914 (2009) [Erratum-ibid. C 81, 029901 (2010)].

[50] E. Frodermann and U. Heinz, Phys. Rev. C 80, 044903 (2009).

[51] M. M. Aggarwal et al. [ WA98 Collaboration], Phys. Rev. Lett. 93, 022301 (2004).

[52] S. A. Bass, B. Muller, and D. K. Srivastava, Phys. Rev. Lett. 90, 082301 (2003), S. A. Bass, B. Muller, and D. K. Srivastava, Phys. Rev. C 66, 061902 (2002); S. A. Bass, T. Renk, and D. K. Srivastava, Nucl. Phys. A 783, 367 (2007).

[53] R. J. Fries, B. Muller, and D. K. Srivastava, Phys. Rev. Lett. 90, 132301 (2003); R. J. Fries, B. Muller, and D. K. Srivastava, Phys. Rev. C 72, 041902 (2005).

[54] G. Y. Qin, C. Gale, S. Jeon, G. D. Moore and J. Ruppert, Nucl. Phys. A 830, 459C (2009); G. Y. Qin, J. Ruppert, C. Gale, S. Jeon, and G. D. Moore, Phys. Rev. C 80, 054909 (2009); S. Turbide, C. Gale, S. Jeon and G. D. Moore, Phys. Rev. C 72, 014906 (2005).

[55] See e.g., C. Nonaka and S. A. Bass, Phys. Rev. C 75, 014902 (2007).

[56] R. Chatterjee, D. K. Srivastava, U. W. Heinz, and C. Gale, Phys. Rev. C 75, 054909 (2007); U. W. Heinz, R. Chatterjee, E. S. Frodermann, C. Gale, and D. K. Srivastava, Nucl. Phys. A 783, 379 (2007). 